\title{
CdSe/CdMnS Nanoplatelets with Bilayer Core and Magnetically Doped Shell Exhibit Switchable Excitonic Circular Polarization: Implications for Lasers and Light-Emitting Diodes
}

Arman Najafi," Steven Tarasek," Savas Delikanli, Peiyao Zhang, Tenzin Norden, Sushant Shendre, Manoj Sharma, Arinjoy Bhattacharya, Nima Taghipour, James Pientka, Hilmi Volkan Demir, Athos Petrou, and Tim Thomay*

Cite This: ACS Appl. Nano Mater. 2020, 3, 3151-3156

Read Online

ABSTRACT: We utilized time-resolved photoluminescence (TRPL) spectroscopy to study the excitonic circular polarization $\left(P_{\mathrm{X}}\right)$ from $\mathrm{CdSe} / \mathrm{CdMnS}$ core/shell nanoplatelets (NPLs) with a bilayer core. This allows an extensive study of the emission dynamics as a function of magnetic field, temperature, doping concentration, and excitation wavelength. In the presence of an external magnetic field, pulsed excitation below the shell gap results in near-zero excitonic circular polarization $P_{\mathrm{X}}$ at all time delays. In contrast, pulsed excitation with photon energy larger than the shell gap results in a rapid (100 ps) buildup of the excitonic circular polarization which subsequently remains constant at a level of up to $40 \%$. We propose a model to describe the dynamics which takes into account the exchange

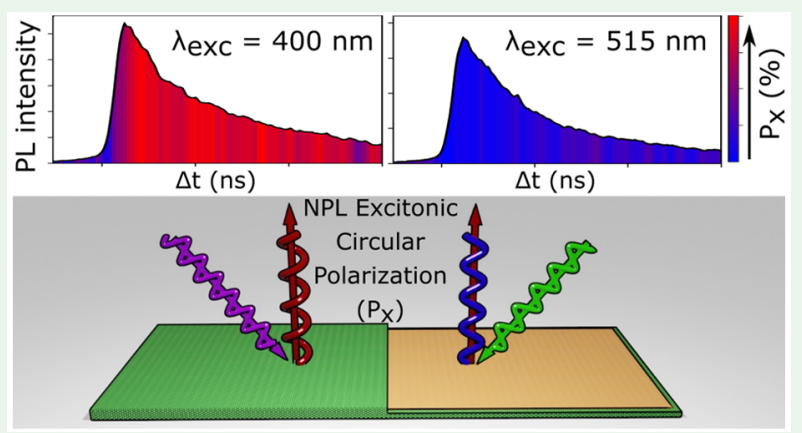
interaction between carrier and magnetic ion $(\mathrm{Mn})$ spins. The studied system exhibits a fast switchable excitonic circular polarization, implying possible applications in lasers and light emitting diodes.

KEYWORDS: nanoplatelets, quasi-2D, time-resolved photoluminescence, spd exchange interaction, magneto-optical switch

Cemiconductor nanoplatelets (NPLs) have been continuing $\checkmark$ to garner attention due to their narrow emission spectra, ${ }^{1-5}$ potential for tunable emission, ${ }^{4,6,7}$ and atomically controlled thicknesses leading to quasi-2D structures ${ }^{8-11}$ with very high quantum efficiency (QE)., ${ }^{2,9,12,13}$ NPLs have emerged as a promising platform for optoelectronic devices such as light emitting diodes (LEDs) $)^{4,14-17}$ and lasers. ${ }^{3,7,9,14,18}$ Recently, it has been demonstrated that the presence of charged excitons in NPLs ${ }^{19,20}$ or doping with magnetic ions such as Mn either in the core or in the shell gives these structures novel magnetooptical properties. $^{21-25}$ A magneto-optical study of NPLs is a particularly powerful tool for the exploration and understanding of their properties. This is realized by using the $\mathrm{Mn}$ ions as a "tracer", which allows the study of the interaction of their spins with spins of the carriers. This provides the necessary information on the static and dynamic behaviors of the system, which is required for the design and performance evaluation of NPL-based devices.

In this work, we study a magnetically doped CdSe/CdMnS core/shell NPL system with a significantly thinner core of only 2 monolayers (bilayer) compared to our previous works. ${ }^{21,22}$ In the presence of an external magnetic field, these NPLs exhibit circularly polarized emission that is dependent on the excitation wavelength. This gives the ability to switch between low and high values of circularly polarized excitonic luminescence, which is a crucial feature for spin polarized lasers and LEDs. ${ }^{26}$ Previous measurements on similar magnetic core/shell structures with thicker cores showed that an externally applied magnetic field causes a net circular polarization in the emission and that the degree of circular polarization saturates for magnetic fields above $3 \mathrm{~T}^{21}$ The analysis of the photoluminescence (PL) from the thicker core NPLs showed the presence of two distinct spectral features, one corresponding to the excitonic emission of the system (observed in both PL and absorption) and a second feature associated with defect interface states that contributes to the asymmetry of the emission. The maximum in circular polarization occurred at the energy of the PL component associated with the interface states. ${ }^{22}$ However, we point out that in the bilayer core NPLs studied in this work, the circular polarization has a maximum at the exciton energy, and we

Received: February 7, 2020

Accepted: March 30, 2020

Published: March 30, 2020 
(a)

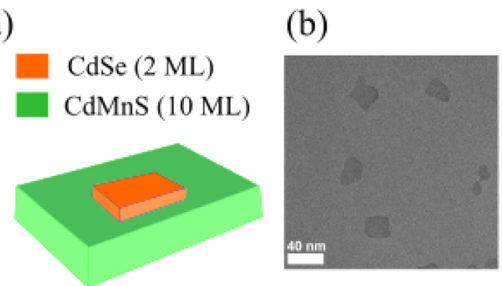

(c)

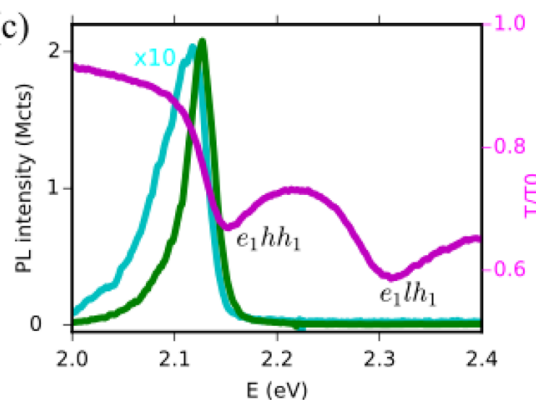

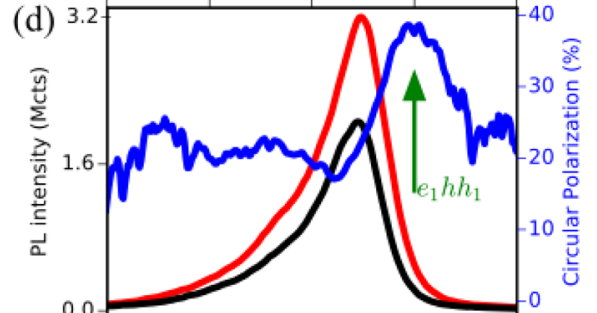

(e)

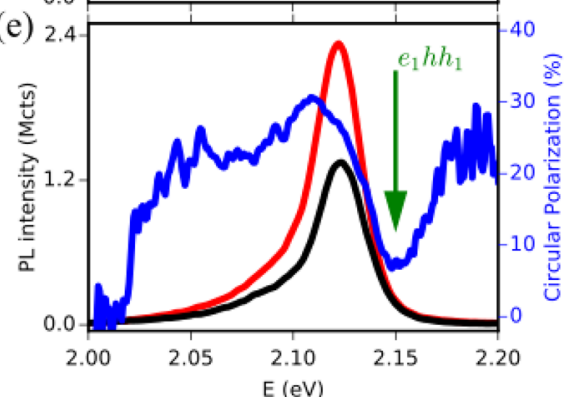

Figure 1. (a) Schematic diagram of the CdSe/CdMnS core/shell NPL samples used in this study. (b) TEM image of a NPL sample. (c) Magenta line depicts the optical transmission spectrum from the high Mn sample; green line: time slice of the TRPL spectrum at early times; cyan line: at later times. (d, e) Time-integrated PL analyzed as $\sigma_{+}$(red) and $\sigma_{-}$(black) and circular polarization (blue) as a function of photon energy at $B=4 \mathrm{~T}$ with excitation under (d) $400 \mathrm{~nm}$ and (e) $515 \mathrm{~nm}$ linearly polarized pulsed laser.

show that the circular polarization of this recombination channel can be controlled by varying the excitation photon energy. We investigated the time evolution of the excitonic circular polarization $P_{\mathrm{X}}$ in the presence of an external magnetic field at $T=7 \mathrm{~K}$. For excitation above the CdMnS shell gap, we observe strong excitonic circular polarization $\left(P_{X}=40 \%\right)$; in contrast, direct $\mathrm{CdSe}$ core excitation results in near-zero excitonic circular polarization $\left(P_{\mathrm{X}} \approx 0 \%\right)$. For above shell gap excitation, a fast (100 ps) switch from near-zero polarization to $40 \%$ is observed.

In Figure 1c, we plot the zero magnetic field transmission spectrum (magenta) from the high Mn concentration NPLs recorded at $T=7 \mathrm{~K}$ along with the PL at early times (green line, at $\Delta t=0 \mathrm{~ns}$ ) and late times (cyan line, at $\Delta t=3.5 \mathrm{~ns}$ ) excited using $400 \mathrm{~nm}$ pulses. The two features at 2.15 and 2.30 $\mathrm{eV}$ of the transmission spectrum are due to the $\mathrm{e}_{1} \mathrm{hh}_{1}$ and $\mathrm{e}_{1} \mathrm{lh}_{1}$ heavy hole and light hole excitonic transitions, respectively. ${ }^{23}$ The $e_{1} h h_{1}\left(e_{1} l h_{1}\right)$ exciton is formed by an electron occupying the first confinement sub-band $\mathrm{e}_{1}$ and a hole in the first heavy hole confinement sub-band $\mathrm{hh}_{1}$ (light hole confinement subband $\mathrm{lh}_{1}$ ). For the $\mathrm{PL}$ at early times, the emission peak coincides with the $\mathrm{e}_{1} \mathrm{hh}_{1}$ exciton, whereas at later times, the emission mostly contains components with energies below $\mathrm{e}_{1} \mathrm{hh}_{1}$; these are attributed to long-lived emission associated with interface states. This separation in time is possible due to the different lifetimes of the excitonic and interface emissions. $^{22}$

In Figures $1 \mathrm{~d}$ and 1e, we plot the left circularly polarized $\sigma_{+}$ (red line) and right circularly polarized $\sigma_{-}$(black line) components of the time-integrated ( $0-5 \mathrm{~ns}) \mathrm{PL}$ at $B=4 \mathrm{~T}$ which are excited using 400 and $515 \mathrm{~nm}$ pulses, respectively. The blue line in these figures represents the resulting circular polarization. Even though the PL spectra in Figure 1d and 1e look similar, the corresponding excitonic circular polarization, identified by the vertical green arrows, is quite distinct. Under $400 \mathrm{~nm}$ excitation (Figure 1d), the polarization has a maximum of $40 \%$ centered at the energy of $\mathrm{e}_{1} \mathrm{hh}_{1}$; however, under $515 \mathrm{~nm}$ excitation (Figure 1e), the excitonic circular polarization $P_{\mathrm{X}}$ is vanishing and the maximum of circular polarization of $30 \%$ coincides with the interface luminescence feature.

Figure 2 shows the magnetic field (inset, $T=7 \mathrm{~K}$ ) and temperature (main figure, $B=4 \mathrm{~T}$ ) dependence of the

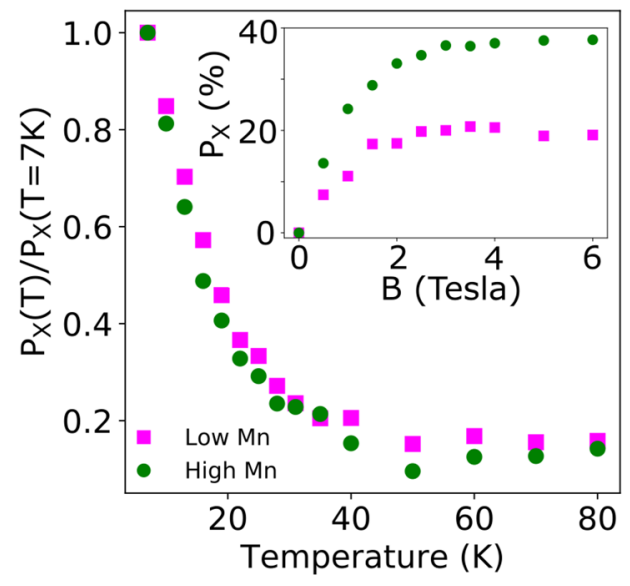

Figure 2. Main: Excitonic circular polarization ratios $P_{\mathrm{X}}(T) / P_{\mathrm{X}}(7 \mathrm{~K})$ plotted as a function of temperature at $B=4 \mathrm{~T}$ and with $400 \mathrm{~nm}$ excitation. Inset: Excitonic circular polarization $P_{\mathrm{X}}$ as a function of magnetic field with $400 \mathrm{~nm}$ excitation. Green circles and magenta squares refer to high and low $\mathrm{Mn}$ concentration samples, respectively.

excitonic circular polarization $P_{\mathrm{X}}$ under $400 \mathrm{~nm}$ excitation. The $P_{\mathrm{X}}$ in the inset increases monotonically with $B$ and saturates around $B=3 \mathrm{~T}$ at 40 and $20 \%$ for the high $\mathrm{Mn}$ and low $\mathrm{Mn}$ doped NPLs, respectively. The ratio $P_{\mathrm{X}}(T) / P_{\mathrm{X}}(7 \mathrm{~K})$ at $B=4 \mathrm{~T}$ plotted in the main figure shows a significant decrease with increasing $T$. The $B$ and $T$ dependence of the excitonic circular polarization $P_{\mathrm{X}}$ demonstrates that the shell exhibits Brillouin Paramagnetic behavior. This leads to the observed $P_{\mathrm{X}}$, which is due to band splittings, where the splittings are proportional to the shell magnetization. The results of Figure 2 provide a strong indication on the existence of sp-d exchange interaction 
between the spins of the electrons (s-symmetry) and holes (psymmetry) and the spins of the $\mathrm{Mn}$ ions (in the $\mathrm{d}$ orbital) in the CdMnS shell. ${ }^{27}$

In Figure $3 \mathrm{a}$ (and $3 \mathrm{~b}$ ) we plot the TRPL intensity sum $I_{\mathrm{PL}}=$ $I_{+}+I_{-}$of the $\sigma_{+}$and $\sigma_{-}$components for the $\mathrm{e}_{1} \mathrm{hh}_{1}$ excitonic

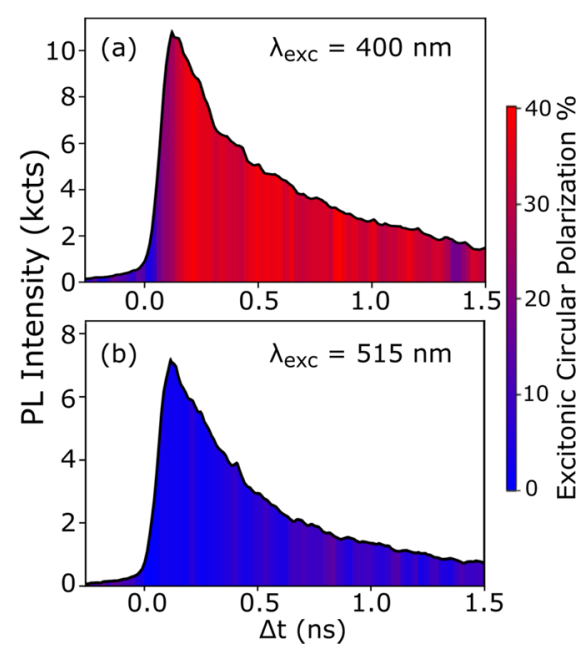

Figure 3. TRPL intensity sum $I_{\mathrm{PL}}=I_{+}+I_{-}$for the high $\mathrm{Mn}$ concentration sample; $B=4 \mathrm{~T} ; T=7 \mathrm{~K}$ (a) under $400 \mathrm{~nm}$ pulsed excitation and (b) under $515 \mathrm{~nm}$ pulsed excitation. The corresponding color scale under the plots represents the circular polarization of the $\mathrm{e}_{1} \mathrm{hh}_{1}$ exciton $\left(P_{\mathrm{X}}\right)$ for each delay time.

feature, indicated by vertical arrows in Figure 1d and 1e, under $400 \mathrm{~nm}(515 \mathrm{~nm})$ pulsed excitation for the high $\mathrm{Mn}$ concentration sample. A single exponential least-square fit (not shown) yields comparable excitonic lifetimes of $0.6 \mathrm{~ns}$ for excitation at $400 \mathrm{~nm}$ and $0.5 \mathrm{~ns}$ for $515 \mathrm{~nm}$ excitation at $B=4$ T. The excitonic circular polarization $P_{\mathrm{X}}$ at a specific time delay is indicated by the color below the $I_{\mathrm{PL}}$ curve (black line), where the area underneath for a particular time slice is proportional to the total number of $\sigma_{+}$and $\sigma_{-}$emitted photons. Figure 3a and $3 \mathrm{~b}$ shows that the $P_{\mathrm{X}}$ depends critically on the excitation photon energy. Under $515 \mathrm{~nm}$ excitation (Figure 3b), the polarization is low at all times. In contrast, using $400 \mathrm{~nm}$ excitation, $P_{\mathrm{X}}$ increases sharply during the rise time (100 ps) of $I_{\mathrm{PL}}$. During this time interval, a significant number of photons with near-zero circular polarization is emitted. For $\Delta t>100 \mathrm{ps}$, $P_{\mathrm{X}}$ reaches a value of $40 \%$ and remains approximately constant. Though we show this for only a single magnetic field $(B=4 \mathrm{~T})$ and the high $\mathrm{Mn}$ concentration sample, we note that this behavior is exhibited for all magnetic field values greater than 3 $\mathrm{T}$ as well as for the low Mn concentration sample.

In this section, we discuss a possible mechanism that is responsible for the observed excitonic circular polarization dependence on excitation photon energy. The electron and hole wave functions in these structures play a crucial role in our model. We show calculated wave functions for the $\mathrm{e}_{1}, \mathrm{hh}_{1}$, and $\mathrm{hh}_{2}$ states in the Supporting Information (Figure S2 and Figure S3). In II-VI diluted magnetic semiconductors, the exchange interaction between the hole and Mn spins is several times larger than the interaction between the electron and $\mathrm{Mn}$ spins, ${ }^{27}$ allowing us to focus on the influence of the external magnetic field on the holes. The five panels in Figure 4 illustrate the dynamics of the excitonic circular polarization $P_{\mathrm{X}}$ according to the proposed model. Panels (a), (b), and (c) refer to an excitation above the core and shell band gaps $(400 \mathrm{~nm})$; panels (d) and (e) refer to $515 \mathrm{~nm}$ excitation which has a photon energy below the shell but above the core band gap.

In panel (a), we indicate the photogeneration of electronhole pairs at $\Delta t=0$ by the incoming pulse. These holes populate excited hole sub-bands that have wave functions which extend throughout the magnetic CdMnS shell (Figure S2). In panel (b), we show the processes for $0<\Delta t<100 \mathrm{ps;}$ during this time holes relax from their excited states to the $\mathrm{hh}_{1}$ ground state (step 2). During the first 100 ps, even though holes are delocalized, their spins do not have time to align antiferromagnetically with $\mathrm{Mn}$ spins that are aligned by the external magnetic field. ${ }^{28,29}$ Therefore, the photons from the $\mathrm{e}_{1} \mathrm{hh}_{1}$ recombination (step 3) have near-zero $P_{\mathrm{X}}$. In panel (c), we show the processes for $\Delta t>100$ ps. During this time, the delocalized holes which had enough time to align their spins antiferromagnetically with the Mn spins, are predominately in the $+3 / 2$ state. We assume that during nonradiative relaxation of the holes to the $\mathrm{hh}_{1}$ ground state, their spin state is at least partially conserved. Now that the majority of holes are in the $+3 / 2$ state, the emitted photons (step 3 ) are circularly polarized as $\sigma_{+}$due to the fact that we have more $-1 / 2$ to $+3 / 2$ than $+1 / 2$ to $-3 / 2$ recombinations. We note that this picture is in agreement with the data shown in Figure $3 \mathrm{a}$.

In panel (d), we show the electron-hole pair generation under $515 \mathrm{~nm}$ excitation. In this case, the holes are strongly confined in the core and interact with the Mn only via the tail of the hole wave function that penetrates into the magnetic shell (Figure S3). In panel (e), we show the hole relaxation to the $\mathrm{hh}_{1}$ state (step 2). The difference in population between the $+3 / 2$ and $-3 / 2$ states is small; therefore, the emitted photons (step 3 ) have a near-zero $P_{\mathrm{X}}$ in agreement with the results of Figure $3 \mathrm{~b}$.

We investigated the time evolution of the circular polarization $P_{\mathrm{X}}$ for the $\mathrm{e}_{1} \mathrm{hh}_{1}$ exciton from CdSe/CdMnS core/shell NPLs with bilayer cores doped with $\mathrm{Mn}$ in the shell. In the presence of a magnetic field, the excitonic circular polarization $P_{\mathrm{X}}$ is strongly dependent on the excitation photon energy. For photons with an energy smaller than the CdMnS shell gap but bigger than the $\mathrm{CdSe}$ core gap, the excitonic circular polarization $P_{\mathrm{X}}$ remains low for all times. However, excitonic circular polarization $P_{\mathrm{X}}$ using an excitation with energy above the CdMnS shell gap results in $40 \%$ circular polarization of the $\mathrm{e}_{1} \mathrm{hh}_{1}$ excitonic ground state emission. In our time-resolved experiments, $P_{\mathrm{X}}$ builds up from a low value following excitation to a constant value within 100 ps. We developed a model that explains this behavior which takes into account the time required by the hole spins in the shell to align with the $\mathrm{Mn}$ spins. Such a system demonstrates the ability to switch the excitonic circular polarization depending on the excitation wavelength; this has potential applications in lasers and LEDs. The switching rate would be limited by the lifetime of the excitonic ground state which is in the order of a nanosecond. However, in utilizing the intrinsic switching time of $100 \mathrm{ps}$ of the circular polarization under above shell gap excitation, a switching rate of tens of $\mathrm{GHz}$ would be feasible which could have applications in the fields of circularly polarized filters and polarizers. $^{30,31}$

\section{METHODS}

A schematic of the NPLs in this study is shown in Figure 1a. They are comprised of an undoped CdSe core $(2 \mathrm{ML})$ surrounded by a $\mathrm{Mn}$ doped CdS shell (10 ML) with two different Mn concentrations, 3\% (high) and 1\% (low). The low temperature band gaps of CdSe core 


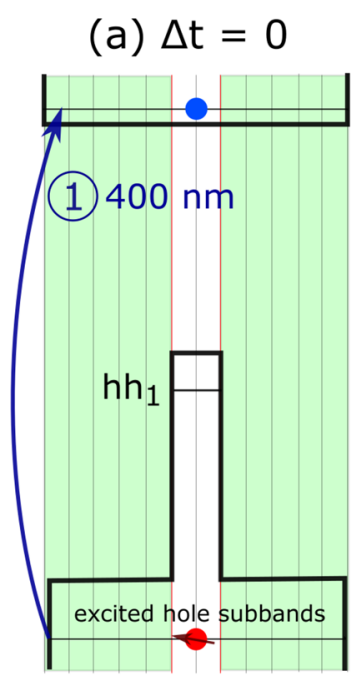

(b) 0

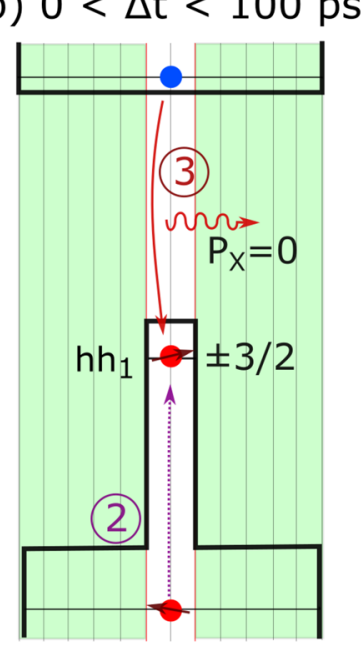

(c) $\Delta \mathrm{t}>100 \mathrm{ps}$

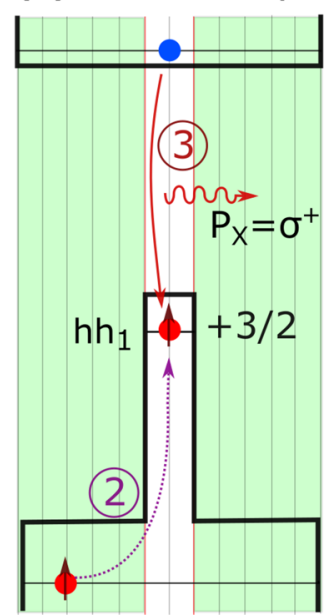

(d) $\Delta \mathrm{t}=0$

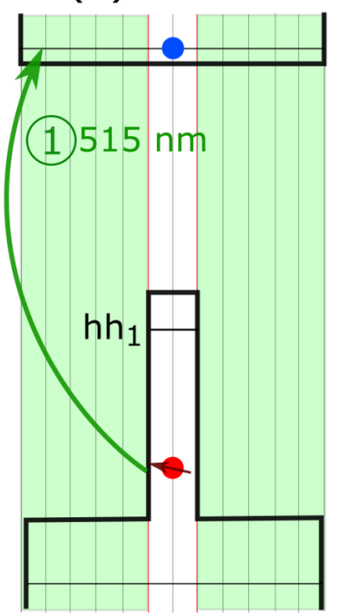

(e) $\Delta \mathrm{t}>0 \mathrm{ps}$

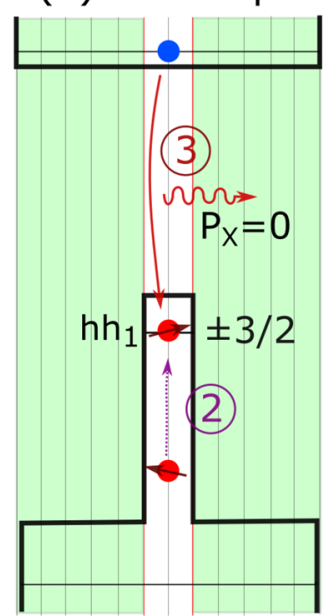

Figure 4. Schematic of the excitonic emission process. (a) Photoexcitation with the $400 \mathrm{~nm}$ pulse at $\Delta t=0$, (b) relaxation/recombination processes for $0<\Delta t<100 \mathrm{ps}$, (c) relaxation/recombination processes for $\Delta t>100 \mathrm{ps,} \mathrm{(d)} \mathrm{photoexcitation} \mathrm{with} \mathrm{the} 515 \mathrm{~nm}$ pulse at $\Delta t=0$, and (e) relaxation/recombination processes for all times $\Delta t>0 \mathrm{ps}$. The red arrows indicate the direction of the hole spin assuming that the magnetic field points up.

and $\mathrm{CdMnS}$ shell are 1.84 and $2.56 \mathrm{eV}$, respectively. ${ }^{23}$ The samples were synthesized by slightly modifying a previously published procedure. $^{23,32}$ A TEM image of the Mn doped core/shell NPLs is presented in Figure 1b, showing lateral sizes of the NPLs on the order of $35 \mathrm{~nm}$. Analysis of the small angle electron diffraction (SAED) pattern presented in Figure S1 in the Supporting Information shows that our Mn doped core/shell NPLs have the zinc-blend structure.

Solution based NPLs were drop-cast either on silicon or sapphire substrates. For magneto-optical experiments, the samples were placed in a variable temperature optical cryostat equipped with a $7 \mathrm{~T}$ superconducting magnet. The measurements were performed in the Faraday geometry, with the direction of the applied field being parallel to the direction of the emitted light propagation. The transmission measurements were performed using a collimated white light beam from a tungsten-halogen lamp, with the transmitted light being collected and analyzed by a grating spectrometer equipped with a cooled CCD detector. The time-resolved photoluminescence (TRPL) was excited using a pulsed laser system with wavelengths of 400 and $515 \mathrm{~nm}$, having a repetition rate of $250 \mathrm{kHz}$, a pulse width of $<200 \mathrm{fs}$, and a pulse energy of approximately $4 \mu \mathrm{J}$. The linearly polarized 400 $\mathrm{nm}$ pulse was generated through second harmonic generation (SHG), while the linearly polarized $515 \mathrm{~nm}$ pulse was generated through an optical parametric amplification process. The NPLs emission was collected and spectrally/temporally analyzed using a spectrometer/ streak camera combination with a temporal resolution of $30 \mathrm{ps}$.
The time-resolved $\sigma_{+}$and $\sigma_{-}$PL components were separated using a combination of a quarter wave plate and a linear polarizer before the spectrometer/streak camera entrance slit. The degree of circular polarization at particular photon energy is defined as $P=\left(I_{+}-I_{-}\right) /\left(I_{+}\right.$ $\left.+I_{-}\right)$where $I_{+}\left(I_{-}\right)$is the intensity of the $\sigma_{+}\left(\sigma_{-}\right)$component.

\section{ASSOCIATED CONTENT}

Supporting Information

The Supporting Information is available free of charge at https://pubs.acs.org/doi/10.1021/acsanm.0c00365.

Chemicals used, NPL sample synthesis/growth details, Figure S1: SAED of NPLs, Figure S2: wave function calculation for the electron ground state $e_{1} /$ heavy hole excited state $\mathrm{hh}_{2}$, Figure S3: wave function calculations for the electron ground state $e_{1}$ /heavy hole ground state $\mathrm{hh}_{1}$ (PDF)

\section{AUTHOR INFORMATION}

\section{Corresponding Author}

Tim Thomay - Department of Physics, State University of New York at Buffalo, Buffalo, New York 14260, United States; 
ำ orcid.org/0000-0003-2271-6803; Email: thomay@ buffalo.edu

\section{Authors}

Arman Najafi - Department of Physics, State University of New York at Buffalo, Buffalo, New York 14260, United States

Steven Tarasek - Department of Physics, State University of New York at Buffalo, Buffalo, New York 14260, United States Savas Delikanli - Luminous! Centre of Excellence for Semiconductor Lighting and Displays, School of Electrical and Electronic Engineering, School of Physical and Mathematical Sciences, School of Materials Science and Engineering, Nanyang Technological University, Singapore 639798 Singapore; Department of Electrical and Electronics Engineering, Department of Physics, UNAM-Institute of Materials Science and Nanotechnology, Bilkent University, Ankara 06800, Turkey

Peiyao Zhang - Department of Physics, State University of New York at Buffalo, Buffalo, New York 14260, United States

Tenzin Norden - Department of Physics, State University of New York at Buffalo, Buffalo, New York 14260, United States Sushant Shendre - Luminous! Centre of Excellence for Semiconductor Lighting and Displays, School of Electrical and Electronic Engineering, School of Physical and Mathematical Sciences, School of Materials Science and Engineering, Nanyang Technological University, Singapore 639798 Singapore

Manoj Sharma - Luminous! Centre of Excellence for Semiconductor Lighting and Displays, School of Electrical and Electronic Engineering, School of Physical and Mathematical Sciences, School of Materials Science and Engineering, Nanyang Technological University, Singapore 639798 Singapore; Department of Electrical and Electronics Engineering, Department of Physics, UNAM-Institute of Materials Science and Nanotechnology, Bilkent University, Ankara 06800, Turkey; O orcid.org/0000-0001-5215-9740

Arinjoy Bhattacharya - Department of Physics, State University of New York at Buffalo, Buffalo, New York 14260, United States

Nima Taghipour - Department of Electrical and Electronics Engineering, Department of Physics, UNAM-Institute of Materials Science and Nanotechnology, Bilkent University, Ankara 06800, Turkey

James Pientka - Department of Physics, St. Bonaventure University, St. Bonaventure, New York 14778, United States; ๑ orcid.org/0000-0003-3167-4246

Hilmi Volkan Demir - Luminous! Centre of Excellence for Semiconductor Lighting and Displays, School of Electrical and Electronic Engineering, School of Physical and Mathematical Sciences, School of Materials Science and Engineering, Nanyang Technological University, Singapore 639798 Singapore; Department of Electrical and Electronics Engineering, Department of Physics, UNAM-Institute of Materials Science and Nanotechnology, Bilkent University, Ankara 06800, Turkey; (1) orcid.org/0000-0003-1793-112X

Athos Petrou - Department of Physics, State University of New York at Buffalo, Buffalo, New York 14260, United States

Complete contact information is available at:

https://pubs.acs.org/10.1021/acsanm.0c00365

\section{Author Contributions}

"A.N. and S.T. contributed equally to this work. Notes

The authors declare no competing financial interest.

\section{REFERENCES}

(1) Ithurria, S.; Tessier, M. D.; Mahler, B.; Lobo, R. P. S. M.; Dubertret, B.; Efros, A. L. Colloidal Nanoplatelets with TwoDimensional Electronic Structure. Nat. Mater. 2011, 10, 936-941.

(2) Tessier, M. D.; Javaux, C.; Maksimovic, I.; Loriette, V.; Dubertret, B. Spectroscopy of Single CdSe Nanoplatelets. ACS Nano 2012, 6, 6751-6758.

(3) She, C.; Fedin, I.; Dolzhnikov, D. S.; Demortière, A.; Schaller, R. D.; Pelton, M.; Talapin, D. V. Low-Threshold Stimulated Emission Using Colloidal Quantum Wells. Nano Lett. 2014, 14, 2772-2777.

(4) Fan, F.; Kanjanaboos, P.; Saravanapavanantham, M.; Beauregard, E.; Ingram, G.; Yassitepe, E.; Adachi, M. M.; Voznyy, O.; Johnston, A. K.; Walters, G.; Kim, G.-H.; Lu, Z.-H.; Sargent, E. H. Colloidal CdSe ${ }_{1-x} S_{x}$ Nanoplatelets with Narrow and Continuously-Tunable Electroluminescence. Nano Lett. 2015, 15, 4611-4615.

(5) Shornikova, E. V.; et al. Addressing the Exciton Fine Structure in Colloidal Nanocrystals: The Case of CdSe Nanoplatelets. Nanoscale 2018, 10, 646-656.

(6) Delikanli, S.; Guzelturk, B.; Hernández-Martínez, P. L.; Erdem, T.; Kelestemur, Y.; Olutas, M.; Akgul, M. Z.; Demir, H. V. Continuously Tunable Emission in Inverted Type-I CdS/CdSe Core/Crown Semiconductor Nanoplatelets. Adv. Funct. Mater. 2015, 25, 4282-4289.

(7) She, C.; Fedin, I.; Dolzhnikov, D. S.; Dahlberg, P. D.; Engel, G. S.; Schaller, R. D.; Talapin, D. V. Red, Yellow, Green, and Blue Amplified Spontaneous Emission and Lasing Using Colloidal CdSe Nanoplatelets. ACS Nano 2015, 9, 9475-9485.

(8) Ithurria, S.; Dubertret, B. Quasi 2D Colloidal CdSe Platelets with Thicknesses Controlled at the Atomic Level. J. Am. Chem. Soc. 2008, 130, 16504-16505.

(9) Pedetti, S.; Ithurria, S.; Heuclin, H.; Patriarche, G.; Dubertret, B. Type-II CdSe/CdTe Core/Crown Semiconductor Nanoplatelets. J. Am. Chem. Soc. 2014, 136, 16430-16438.

(10) Nasilowski, M.; Mahler, B.; Lhuillier, E.; Ithurria, S.; Dubertret, B. Two-Dimensional Colloidal Nanocrystals. Chem. Rev. 2016, 116, 10934-10982.

(11) Yoon, D.-E.; Kim, W. D.; Kim, D.; Lee, D.; Koh, S.; Bae, W. K.; Lee, D. C. Origin of Shape-Dependent Fluorescence Polarization from CdSe Nanoplatelets. J. Phys. Chem. C 2017, 121, 24837-24844.

(12) Tessier, M. D.; Mahler, B.; Nadal, B.; Heuclin, H.; Pedetti, S.; Dubertret, B. Spectroscopy of Colloidal Semiconductor Core/Shell Nanoplatelets with High Quantum Yield. Nano Lett. 2013, 13, 33213328.

(13) Shendre, S.; Delikanli, S.; Li, M.; Dede, D.; Pan, Z.; Ha, S. T.; Fu, Y. H.; Hernández-Martínez, P. L.; Yu, J.; Erdem, O.; Kuznetsov, A. I.; Dang, C.; Sum, T. C.; Demir, H. V. Ultrahigh-Efficiency Aqueous Flat Nanocrystals of CdSe/CdS@Cd 1-x Zn ${ }_{x}$ S Colloidal Core/Crown@alloyed-Shell Quantum Wells. Nanoscale 2019, 11, 301-310.

(14) Bose, S.; Song, Z.; Fan, W. J.; Zhang, D. H. Effect of Lateral Size and Thickness on the Electronic Structure and Optical Properties of Quasi Two-Dimensional CdSe and CdS Nanoplatelets. J. Appl. Phys. 2016, 119, 143107.

(15) Cruguel, H.; Livache, C.; Martinez, B.; Pedetti, S.; Pierucci, D.; Izquierdo, E.; Dufour, M.; Ithurria, S.; Aubin, H.; Ouerghi, A.; Lacaze, E.; Silly, M. G.; Dubertret, B.; Lhuillier, E. Electronic Structure of CdSe-ZnS 2D Nanoplatelets. Appl. Phys. Lett. 2017, 110, 152103.

(16) Xiao, P.; Huang, J.; Yan, D.; Luo, D.; Yuan, J.; Liu, B.; Liang, D. Emergence of Nanoplatelet Light-Emitting Diodes. Materials 2018, 11, 1376-1398.

(17) Liu, B.; Delikanli, S.; Gao, Y.; Dede, D.; Gungor, K.; Demir, H. V. Nanocrystal Light-Emitting Diodes Based on Type II Nanoplatelets. Nano Energy 2018, 47, 115-122.

(18) Li, Q.; Xu, Z.; McBride, J. R.; Lian, T. Low Threshold Multiexciton Optical Gain in Colloidal CdSe/CdTe Core/Crown Type-II Nanoplatelet Heterostructures. ACS Nano 2017, 11, 25452553.

(19) Biadala, L.; Liu, F.; Tessier, M. D.; Yakovlev, D. R.; Dubertret, B.; Bayer, M. Recombination Dynamics of Band Edge Excitons in 
Quasi-Two-Dimensional CdSe Nanoplatelets. Nano Lett. 2014, 14, 1134-1139.

(20) Shornikova, E. V.; et al. Electron and Hole $g$-Factors and Spin Dynamics of Negatively Charged Excitons in CdSe/CdS Colloidal Nanoplatelets with Thick Shells. Nano Lett. 2018, 18, 373-380.

(21) Delikanli, S.; Akgul, M. Z.; Murphy, J. R.; Barman, B.; Tsai, Y.; Scrace, T.; Zhang, P.; Bozok, B.; Hernández-Martínez, P. L.; Christodoulides, J.; Cartwright, A. N.; Petrou, A.; Demir, H. V. Mn2+-Doped CdSe/CdS Core/Multishell Colloidal Quantum Wells Enabling Tunable Carrier-Dopant Exchange Interactions. ACS Nano 2015, 9, 12473-12479.

(22) Murphy, J. R.; Delikanli, S.; Scrace, T.; Zhang, P.; Norden, T.; Thomay, T.; Cartwright, A. N.; Demir, H. V.; Petrou, A. TimeResolved Photoluminescence Study of $\mathrm{CdSe} / \mathrm{CdMnS} / \mathrm{CdS}$ Core/ Multi-Shell Nanoplatelets. Appl. Phys. Lett. 2016, 108, 242406.

(23) Muckel, F.; Delikanli, S.; Hernández-Martínez, P. L.; Priesner, T.; Lorenz, S.; Ackermann, J.; Sharma, M.; Demir, H. V.; Bacher, G. $\mathrm{Sp}-\mathrm{d}$ Exchange Interactions in Wave Function Engineered Colloidal CdSe/Mn:CdS Hetero-Nanoplatelets. Nano Lett. 2018, 18, 20472053.

(24) Strassberg, R.; Delikanli, S.; Barak, Y.; Dehnel, J.; Kostadinov, A.; Maikov, G.; Hernandez-Martinez, P. L.; Sharma, M.; Demir, H. V.; Lifshitz, E. Persuasive Evidence for Electron-Nuclear Coupling in Diluted Magnetic Colloidal Nanoplatelets Using Optically Detected Magnetic Resonance Spectroscopy. J. Phys. Chem. Lett. 2019, 10, 4437-4447.

(25) Davis, A. H.; Hofman, E.; Chen, K.; Li, Z.-J.; Khammang, A.; Zamani, H.; Franck, J. M.; Maye, M. M.; Meulenberg, R. W.; Zheng, W. Exciton Energy Shifts and Tunable Dopant Emission in Manganese-Doped Two-Dimensional CdS/ZnS Core/Shell Nanoplatelets. Chem. Mater. 2019, 31, 2516-2523.

(26) Holub, M.; Bhattacharya, P. Spin-Polarized Light-Emitting Diodes and Lasers. J. Phys. D: Appl. Phys. 2007, 40, R179-R203.

(27) Furdyna, J. K. Diluted Magnetic Semiconductors. J. Appl. Phys. 1988, 64, R29-R64.

(28) Schneiber, M.; Seufert, J.; Schoemig, H.; Bacher, G.; Forchel, A. W. B. Spin and Polarization Dynamics in Magnetic and Nonmagentic Semiconductor Quantum Dots. Ultrafast Phenomena in Semiconductors VII; 2003; pp 1-14.

(29) Kłopotowski, Ł.; Cywiński, Ł.; Wojnar, P.; Voliotis, V.; Fronc, K.; Kazimierczuk, T.; Golnik, A.; Ravaro, M.; Grousson, R.; Karczewski, G.; Wojtowicz, T. Magnetic Polaron Formation and Exciton Spin Relaxation in Single CdxMnxTe Quantum Dots. Phys. Rev. B: Condens. Matter Mater. Phys. 2011, 83, 081306.

(30) Kim, B. C.; Lim, Y. J.; Song, J. H.; Lee, J. H.; Jeong, K.-U.; Lee, J. H.; Lee, G.-D.; Lee, S. H. Wideband Antireflective Circular Polarizer Exhibiting a Perfect Dark State in Organic Light-EmittingDiode Display. Opt. Express 2014, 22, A1725-A1730.

(31) Salerno, F.; Berrocal, J. A.; Haedler, A. T.; Zinna, F.; Meijer, E. W.; Bari, L. D. Highly Circularly Polarized Broad-Band Emission from Chiral Naphthalene Diimide-Based Supramolecular Aggregates. J. Mater. Chem. C 2017, 5, 3609-3615.

(32) Delikanli, S.; et al. Ultrathin Highly Luminescent TwoMonolayer Colloidal CdSe Nanoplatelets. Adv. Funct. Mater. 2019, 29, 1901028. 